Vol. 40 (1989) [465-479]

\title{
SUPERCONVERGENCE ANALYSIS OF FLUX COMPUTATIONS FOR NONLINEAR PROBLEMS
}

\author{
S.-S. Chow and R.D. Lazarov
}

\begin{abstract}
In this paper we consider the error estimates for some boundary-flux calculation procedures applied to two-point semilinear and strongly nonlinear elliptic boundary value problems. The case of semilinear parabolic problems is also studied. We prove that the computed flux is superconvergent with second and third order of convergence for linear and quadratic elements respectively. Corresponding estimates for higher order elements may also be obtained by following the general line of argument.
\end{abstract}

\section{INTRODUCTION}

In the application of finite element methods to certain Dirichlet boundary value problems, one is often interested in estimating the flux on the boundary in addition to obtaining an approximation to the solution of the boundary value problem. A simple but effective post-processing procedure was proposed by Wheeler [18] for computing the flux from the finite element approximation of a two-point boundary value problem. By multiplying the governing equation of interest by a test function that does not vanish at both boundary points and performing an integration by parts, an equation for the flux is obtained. The finite element solution may then be used to replace the weak solution in the equation. With an appropriate choice of test function, one obtains a formula for computing the flux at the boundary points.

$y$ As it turns out, this procedure yields boundary flux values that are superconvergent, that is the error of the approximation flux has an order of convergence that is higher than that of the gradient of the finite element approximations. This observation was shown to be true rigorously for linear problems by Wheeler [20] and Dupont [10]. Extension to the two dimensional case for a rectangular domain was proposed and analysed (again for linear problems) by Douglas, Dupont and Wheeler [9].

A inore computationally efficient approach was proposed by Carey [4], The crucial idea being the choice of a localised test function in the flux formula. This allows generalisation to two dimensional problems without restriction to rectangular domains.

\section{Received 13 January 1989}

This work is supported in part by a visiting fellowship for $S$. Chow at the Centre for Mathematical Analysis, Australian National University, while on a research semester leave provided by the College of Arts and Sciences of the University of Wyoning.

Copyriglıt Clearance Centre, Inc. Serial-fee code: 0004-9729/89 \$A2.00+0.00. 
(Carey et al [5]). Numerical calculations in these studies again indicaled the occurence of superconvergence in the flux computation procedure, even when applied to semilinear and strongly nonlinear problems.

In this paper, we consider the error estimates for these flux calculation procedures. For simplicity of presentation, we will restrict ourselves to two-point semilinear and strongly nonlinear problems. How these results may be extended to higher elements will be indicated. The case of semilinear parabolic problems is also studied. We proved that the flux computed has an error of second and third order accuracy for linear and quadratic elements respectively. For related works, see Barrett and Elliott [2] and Lazarov et al [12]. For a recent survey of the superconvergence phenomena, see Křižek and Neittaanmäki [13].

In the next section we consider semilinear problems with linear finite element solutions, then we study in Section 3 strongly nonlinear problems with linear finite element solutions. In Section 4 we return to the semilinear problems for the case of quadratic elements. In the last section we study parabolic semilinear boundary value problems using results from Sections 2 and 4 . Before proceeding, we first intriduce some notation.

\section{Notation.}

Throughout this paper, $\mathbf{P}_{k}(\Omega)$ denotes the space of polynomials of degree at most $k$ defined on the domain $\Omega$. The Sobolev space $W^{k, p}(\Omega), k \in\{0, \pm 1, \pm 2, \ldots\}, p \in$ $[1, \infty]$, is equipped with the usual norm and seminorm denoted by $\|\cdot\|_{k, p, \Omega}$ and $|\cdot|_{k, p, \Omega}$ respectively. We shall omit the index $p$ when $p=2$, and the index $\Omega$ when $\Omega$ is the unit interval. We write $H^{k}(\Omega)$ for $W^{k, 2}(\Omega)$ and $V$ for $H_{0}^{1}(\Omega)$, the subspace of $H^{1}(\Omega)$ with elements vanishing on the boundary of $\Omega$. Since the distinction should be clear from the context, we use $(\cdot, \cdot)$ to denote both the $L^{2}(\Omega)$ inner product and the $H^{-1}(\Omega) \times H_{0}^{1}(\Omega)$ duality pairing.

The unit interval is partilioned into $N$ subintervals $I_{j}=\left(x_{j-1}, x_{j}\right), j \in 1, \ldots, N$, with $x_{0}=1$ and $x_{N}=1$ and $x_{j-1}<x_{j}$. Let $h$ denote the maximum diameter of the suljintervals. We write $S^{h}$ for the finite element spacing corresponding to the partition (Ciarlet [7]) and $S_{0}^{h}$ for the space $V \cup S^{h}$. The constant $C$ is generic and is independent of the parameter $h$.

The superconvergence phenomenon only occurs when the solution to the boundary value problem is sufficiently smooth. Thus we assume in the sequel that $u$ has sufficient regularity for any norm used in the analysis to make sense.

If $S^{h}$ possesses finite elements of degree $r-1$, then for a sufficiently smooth function $v$ we may define the Lagrangian interpolation $v_{I}$ of $v$ in $S^{h}$. Furthermore, we have the following error estimate (Strang and Fix [15], Ciarlet [7]):

$$
\left\|v-v_{I}\right\|_{0, \Omega} \leqslant C h^{r}\|v\|_{r, \Omega} .
$$




\section{Semilinear problems With Linear elements}

Let us first consider the application of the flux computation procedure to the semilinear problem

$$
\begin{gathered}
-\left(a(x) u^{\prime}(x)\right)^{\prime}=f(u) \text { in } \Omega \equiv(0,1) \\
u(0)=\alpha, u(1)=\beta
\end{gathered}
$$

where $\alpha$ and $\beta$ are constants. Here we assume that $a \in W^{1, \infty}(\Omega)$ and is bounded below and above by some positive constants $a_{0}, a_{1}$ in $\Omega$, that is

$$
0<a_{0} \leqslant a(x) \leqslant a_{1}<\infty \text { a.e. in } \Omega
$$

The function $f(\cdot)$ is a monotonically decreasing, Lipschitz continuous function such that for all $v, w$ in $V$,

$$
(f(w)-f(v), w-v) \leqslant \gamma\|w-v\|_{0}^{2}
$$

We further assume that for all $w, v$ in $V$, there exists a constant $\delta>0$ such that

$$
a_{0}|w-v|_{1}^{2}-\gamma\|w-v\|_{0}^{2} \geqslant \delta|w-v|_{1}^{2}
$$

This condition limits the size of $\gamma$ and guarantees the strict convexity of the associated energy functional, thus ensuring the existence and uniqueness of the solution to the following weak problern:

$$
a(u, v)=(f(u), v) \text { for all } v \text { in } V
$$

where $a(u, v)=\left(a(x) u^{\prime}(x), v^{\prime}(x)\right)$.

We define the finite element approximation $u_{h}$ of $u$ via

$$
a\left(u_{h}, v_{h}\right)=\left(f\left(u_{h}\right), v_{h}\right) \text { for all } v_{h} \text { in } S_{0}^{h}
$$

From standard finite element theory (Douglas and Dupont [8], Noor and Whiteman [14]), we have the error bound

$$
\left\|u-u_{h}\right\|_{0} \leqslant C h^{r}\|u\|_{r}
$$

Once the finite element approximation $u_{h}$ is available, we may proceed to compute the approximate flux using the integration by parts formula. Recall that the true flux $q \equiv-a u^{\prime}$ satisfies the equation

$$
-\left.q v\right|_{0} ^{1}=a(u, v)-(f(u), v) \text { for all } v \text { in } H^{1}(\Omega)
$$


Thus we approximate $q$ by $q_{h}$, by evaluating

$$
-\left.q_{h} v_{h}\right|_{0} ^{1}=a\left(u_{h}, v_{h}\right)-\left(f\left(u_{h}\right), v_{h}\right) \text { for all } v_{h} \text { in } v^{h}
$$

In order to estimate the error $\left|\left(q-q_{h}\right)(0)\right|+\left|\left(q-q_{h}\right)(1)\right|$, we subtract (9) from (8) and introduce the interpolant $u_{1}$ of $u$ in $S^{h}$ to get

$$
\begin{gathered}
\left.\left(q_{h}-q\right) v_{h}\right|_{0} ^{1}=a\left(u-v_{1}, v_{h}\right)+a\left(u_{I}-u_{h}, v_{h}\right)+\left(f\left(u_{h}\right)-f(u), v_{h}\right) \\
\text { for all } v_{h} \text { in } S^{h} .
\end{gathered}
$$

Restricting ourselves for now to the special case of linear elements, so $r=2$ in (7), and assuming $u$ is sufficiently smooth, we now estimate each of the three terms on the right hand side of (10) and show that they are bounded by an $O\left(h^{2}\right)$ term.

For the last term in (10), since $f$ is Lipschitz continuous, we have

$$
\begin{aligned}
\left|\left(f\left(u_{h}\right)-f(u), v_{h}\right)\right| & \leqslant \int_{\Omega}\left|f\left(u_{h}\right)-f(u)\right|\left|v_{h}\right| d x \\
& \leqslant C \int_{\Omega}\left|u_{h}-u\right|\left|v_{h}\right| d x \\
& \leqslant C\left\|u_{h}-u\right\|_{0}\left\|v_{h}\right\|_{0} \\
& \leqslant C h^{2}\|u\|_{2}\left\|v_{h}\right\|_{0} .
\end{aligned}
$$

To estimate the first term, we write it as a sum of integrals over $I_{j}$ and apply integration by parts, while noting that $v_{h}^{\prime}$ is constant over each $I_{j}$ and that $u_{I}$ agrees with $u$ at the nodes $x_{j}$. Thus using (1) we have

$$
\begin{aligned}
\left|a\left(u-u_{1}, v_{h}\right)\right| & =\left|\sum_{j=1}^{N} \int_{x_{j-1}}^{x_{j}} a\left(u-u_{I}\right)^{\prime} v_{h}^{\prime} d x\right| \\
& \leqslant \sum_{j=1}^{N}\left|v_{h}^{\prime}\right|\left|a^{\prime}\left(u-u_{I}\right)\right|_{x_{j-1}}^{x_{j}}-\int_{x_{j-1}}^{x_{j}} a^{\prime}\left(u-u_{I}\right) d x \\
& \leqslant \int_{0}^{1}\left|a^{\prime}\right|\left|u-u_{I}\right|\left|v_{h}^{\prime}\right| d x \\
& \leqslant\|a\|_{1, \infty}\left\|u-u_{I}\right\|_{0}\left|v_{h}\right|_{1} \\
& \leqslant C h^{2}\|u\|_{2}\left|v_{h}\right|_{1} .
\end{aligned}
$$

Before estimating the remaining term, we note that in view of (3), the bilinear form $a(\cdot, \cdot)$ is coercive and continuous. Also from (5) and (6) we have for all $v_{h}$ in $S_{0}^{h}$,

$$
a\left(u_{h}, v_{h}\right)=a\left(u, v_{h}\right)-\left(f(u)-f\left(u_{h}\right), v_{h}\right)
$$


Thus applying (11) and (12), and noting that $u_{I}-u_{h}$ is in $S_{0}^{h}$,

$$
\begin{aligned}
C\left\|u_{I}-u_{h}\right\|_{1}^{2} & \leqslant a\left(u_{I}-u_{h}, u_{I}-u_{h}\right) \\
& =a\left(u_{I}-u, u_{I}-u_{h}\right)+\left(f(u)-f\left(u_{h}\right), u_{I}-u_{h}\right) \\
& \leqslant C h^{2}\|u\|_{2}\left|u_{I}-u_{h}\right|_{1}+C h^{2}\|u\|_{2}\left\|u_{I}-u_{h}\right\|_{0}
\end{aligned}
$$

and thus

$$
\left|u_{I}-u_{h}\right|_{1} \leqslant\left\|u_{I}-u_{h}\right\|_{1} \leqslant C h^{2}\|u\|_{2} .
$$

Cousequently,

$$
\left|a\left(u_{I}-u_{h}, v_{h}\right)\right| \leqslant C\left|u_{I}-u_{h}\right|_{1}\left|v_{h}\right|_{1} \leqslant C h^{2}\|u\|_{2}\left|v_{h}\right|_{1} .
$$

Note that the inequality (13) is in itself a superconvergence result, well-known for linear problems. It establishes the fact that $u_{I}$ and $u_{h}$, both elements of $S^{h}$, are asymptotically closer to one another than they are to $u$. This interior superconvergence phenomenon is used heavily in estimating the order of convergence of the flux approximations for the nonlinear problems considered here and in two dimensional problems (Larazov et al [12]).

Combining (11), (12) and (14) we obtain

Theonem 1. The approximate flux $q_{h}$ of the semilinear problem (2) using linear elements satisfies the inequality

$$
\left.\left|\left(q-q_{h}\right) v_{h}\right|_{0}^{1}\left|\leqslant C h^{2}\|u\|_{2}\right| v_{h}\right|_{1}
$$

for all $v_{h} \in S^{h}$.

Setting $v_{h}=x$ and $1-x$ respectively as in Wheeler [20], we have

CoRollary 1. The approximate flux corresponding to (2) using linear elements is second order accurate, that is

$$
\left|\left(q-q_{h}\right)(0)\right|+\left|\left(q-q_{h}\right)(1)\right| \leqslant C h^{2}\|u\|_{2} .
$$

Observe that if we set $v_{h}$ to be the piecewise linear finite element basis functions $\varphi_{0}, \varphi_{N}$ associated with the elements on the boundary, as in Carey [4], then since $\left|v_{h}\right|_{1}=O\left(h^{-1 / 2}\right)$, we only have al first sight an $O\left(h^{3 / 2}\right)$ estimate for the flux, which is still superconvergent but suboptimal when compared with the $O\left(h^{2}\right)$ estimate in (15), and is not in agreement with numerical experience. On closer examination, we see that since

$$
a\left(u-u_{h}, w_{h}\right)=0 \text { for all } w_{h} \text { in } S_{0}^{h}
$$


so by setting for example $w_{h}=\varphi_{N}-x$, we may replace the $a\left(u-u_{h}, \varphi_{N}\right)$ term by $a\left(u-u_{h}, x\right)$ to get

$$
\left.\left(q_{h}-q\right) \varphi_{N}\right|_{0} ^{1}=a\left(u-u_{h}, x\right)
$$

which then yields the desired optimal superconvergent result. Similarly we may replace $a\left(u-u_{h}, \varphi_{0}\right)$ by $a\left(u-u_{h}, 1-x\right)$.

\section{Strongly NONLINEAR PRoblems With LINEAR ELEMENTS}

Next we turn to the strongly nonlinear problem

$$
\begin{aligned}
-\left(k\left(\left|u^{\prime}\right|\right) u^{\prime}\right) & =f(x) \text { in } \Omega \equiv(0,1) \\
u(0) & =0, u(1)=0
\end{aligned}
$$

where $k(t)$ is a continuous function of $t \geqslant 0$ with the following properties

$$
\begin{gathered}
m \leqslant k(t),(k(t) t)^{\prime} \leqslant M \text { for all } t \geqslant 0 \\
\left|(k(t) t)^{\prime \prime}\right| \leqslant M_{1} \text { for all } t>0
\end{gathered}
$$

Here $m, M$ and $M_{1}$ denote finite posilive constants.

A typical example is $k(t)=a+c t /(b t+d)$ with $a, b, c, d$ all being positive constants. Problems with this type of nonlinearity occur in many applications, one of which is the design of turbo machinery (Glowinski and Marrocco [11]). The assumed properties of $k$ allow us to study the problem of well-posedness of (16) with the aid of the theory of monotone operators (Vainberg [17], Browder [3]). Error estimates for the finite element approximation may also be derived by exploiting the stated properties of $k$ and the monotonicily and continuity properties of the operator associated with the problem (Glowinski and Marrocco [11], Chow [6]).

Writing $k$ for $k\left(\left|u^{\prime}\right|\right), k_{I}$ for $k\left(\left|u_{I}^{\prime}\right|\right)$ and $k_{h}$ for $k\left(\left|u_{h}^{\prime}\right|\right)$ in this section, where $u$, $u_{I}$ and $u_{h}$ are the weak solution of (16), the piecewise linear interpolant of $u$ in $S^{h}$ and the finite element approximation of $u$ in $S^{h}$ respectively, the flux $q \equiv-k u^{\prime}$ and the approximate flux $q_{h}$ satisfy respectively the equations

and

$$
\begin{aligned}
-\left.q v\right|_{0} ^{1} & =(A u, v)-(f, v) \text { for all } v \text { in } H^{1}(\Omega) \\
& \equiv \int_{0}^{1} k u^{\prime} v^{\prime} d x-\int_{0}^{1} f v d x \\
-\left.q_{h} v_{h}\right|_{0} ^{1} & =\left(A u_{h}, v_{h}\right)-\left(f, v_{h}\right) \text { for all } v_{h} \text { in } S^{h}
\end{aligned}
$$

Subtracting the above equations and introducing $u_{1}$ as before, we get

$$
\left.\left(q_{h}-q\right) v_{h}\right|_{0} ^{1}=\left(A u-A u_{1}, v_{h}\right)+\left(A u_{I}-A u_{h}, v_{h}\right)
$$


Under the regularity assumption that the weak solution $u$ of $(16)$ belongs to $W^{2, \infty}(\Omega)$, we now show that the right hand terms in (18) are of $O\left(h^{2}\right)$ when we take $S^{h}$ to be the linear finite element space.

Applying the mean value theorem, and noting that $v_{h}^{\prime}$ and $u_{I}^{\prime}$ are piecewise constant functions, we have

$$
\begin{aligned}
\left(A u-A u_{I}, v_{h}\right) & =\sum_{j=1}^{N} \int_{x_{j-1}}^{x_{j}}\left(k u^{\prime}-k_{I} u_{I}^{\prime}\right) v_{h}^{\prime} d x \\
& =\sum_{j=1}^{N} v_{h}^{\prime} \int_{x_{j-1}}^{x_{j}} \int_{0}^{1} K(s) d s\left(u^{\prime}-u_{I}^{\prime}\right) d x
\end{aligned}
$$

where

$$
K(s)=\left.(k(|t|) t)^{\prime}\right|_{t=s u^{\prime}+(1-s) u_{I}^{\prime}} .
$$

Since over each subinterval, $u_{I}^{\prime \prime}=0,|(d / d x) K(s)|=\left|K^{\prime}(s) s u^{\prime \prime}\right| \leqslant M_{1} s\left|u^{\prime \prime}\right|$, so proceeding as in the semilinear case, we have

$$
\begin{aligned}
\left|\left(A u-A u_{I}, v_{h}\right)\right| & \leqslant \sum_{j=1}^{N}\left|-v_{h}^{\prime} \int_{x_{j-1}}^{x_{j}} \int_{0}^{1} \frac{d}{d x} K(s) d s\left(u-u_{I}\right) d x\right| \\
& \leqslant \int_{0}^{1}\left(\left|v_{h}^{\prime}\right| \int_{0}^{1} M_{1} s\left|u^{\prime \prime}\right| d s\left|u-u_{I}\right|\right) d x \\
& \leqslant C|u|_{2, \infty}\left|v_{h}\right|_{1}\left\|u-u_{I}\right\|_{0} \\
& \leqslant C h^{2}|u|_{2, \infty}\|u\|_{2}\left|v_{b}\right|_{1} .
\end{aligned}
$$

To estimate the second term we need to utilise the strongly monolone property of the operator $A$ generated by property (i) (Chow [6]). Thus using (19) and the fact that $u_{I}-u_{h}$ is in $S_{0}^{h}$ we get

$$
\begin{aligned}
C\left|u_{I}-u_{h}\right|_{1}^{2} & \leqslant\left(A u_{I}-A u_{h}, u_{I}-u_{h}\right) \\
& =\left(A u_{I}-A u, u_{I}-u_{h}\right) \\
& \leqslant C h^{2}|u|_{2, \infty}\|u\|_{2}\left|u_{I}-u_{h}\right|_{1}
\end{aligned}
$$

and hence we have the interior superconvergence result

$$
\left|u_{I}-u_{h}\right|_{1} \leqslant C h^{2}|u|_{2, \infty}\|u\|_{2} .
$$

Using this result, we may now obtain a bound on the second term using the Lipschitz continuity of $k(t) t$ :

$$
\begin{aligned}
\left|\left(A u_{I}-A u_{h}, v_{h}\right)\right| & \leqslant \int_{0}^{1}\left|k_{I} u_{I}^{\prime}-k_{h} u_{h}^{\prime}\right|\left|v_{h}^{\prime}\right| d x \\
& \leqslant C\left|u_{I}-u_{h}\right|_{1}\left|v_{h}\right|_{1} \\
& \leqslant C h^{2}|u|_{2, \infty}\|u\|_{2}\left|u_{h}\right|_{1} .
\end{aligned}
$$

On combining (19) and (20) we obtain 
Theоrem 2. The approximate flux $q_{h}$ for (16) using linear elements satisfies the estimate

$$
\left.\left|\left(q-q_{h}\right) v_{h}\right|_{0}^{1}\left|\leqslant C h^{2}\right| u\right|_{2, \infty}\|u\|_{2}\left|v_{h}\right|_{1} \text { for all } v_{h} \text { in } S^{h} .
$$

And, setting $v_{h}=x$ and $1-x$ respectively, we arrive at the desired result.

Corollary 2. The approximate flux $q_{h}$ for (16) using linear elements is second order accurate, that is

$$
\left|\left(q-q_{h}\right)(0)\right|+\left|\left(q-q_{h}\right)(1)\right| \leqslant C h^{2}|u|_{2, \infty}\|u\|_{2} .
$$

\section{Semilinear PROBLEMS WITI QUADRATIC ELEMENTS}

Let us now turn our attention to the flux approximation of the semilinear problem (2) in Section 2 using quadratic elements. The result obtained previously relies on the fact that, for linear element, the derivatives of the corresponding test function is piecewise constant. This is certainly not so for quadratic elements. Moreover, interior superconvergence phenomena are often observed at specific points, for example Gauss points, rather than globally over the whole domain as indicated in the linear element case. So we introduce numerical integration in our finite element formulation in the following analysis of the flux approximation.

We shall proceed in much the same manner as in the proof of the linear case, while utilising some ideas outlined in the works of Zlamal [21] and Andreev and Lazarov [1] on the interior superconvergence estimates of the gradient for quadratic elements. Thus we first define an integration scheme $I_{e}(f)$ that is exact for all cubic polynomials $f$, for evaluating the integral $\int_{e} f d x$ :

$$
I_{e}(f)=\sum_{k} w_{k} f\left(x_{k}\right), x_{k} \in e .
$$

For our purpose, we take, as the desired quadrature formula. Note that in this case the coefficients $w_{k}$ are all positive.

We denote by $a_{h}\left(u_{h}, v_{h}\right)$ a bilinear form that approximates $a\left(u_{h}, v_{h}\right)$ under the numerical integration scheme (21):

$$
a_{h}\left(u_{h}, v_{h}\right)=\sum_{j=1}^{N} I_{e_{j}}\left(a(x) u_{h}^{\prime} v_{h}^{\prime}\right)
$$

where $e_{j}$ is the $j$ th finite element over $\left[x_{j-1}, x_{j}\right]$. As it is often more convenient to perform the computation over a reference element defined over $\hat{\Omega} \equiv[-1,1]$, we also write

$$
a_{h}\left(u_{h}, v_{h}\right)=\sum_{j=1}^{N} \hat{I}\left[\hat{a} \frac{d \hat{u}_{h}}{d \xi} \frac{d \hat{v}_{h}}{d \xi}\right]
$$


where $\hat{I}$ denotes the integration scheme (21) applied over $\hat{\Omega}$ with $\hat{w}_{k}=\left(2 / h_{j}\right) w_{k}$. Here $h_{j}$ is the diameter of $e_{j}$ and $x=\left(h_{j} / 2\right)(\xi+1)+x_{j-1}$ for $x \in e_{j}$.

The approximate flux $q_{h}$ is then obtained from

$$
-\left.q_{h} v_{h}\right|_{0} ^{1}=a_{h}\left(u_{h}, v_{h}\right)-\left(f\left(u_{h}\right), v_{h}\right)
$$

Note that we have assumed the semilinear term may be evaluated exactly. The analysis for the case where numerical integration is also applied to this term is not difficult and is therefore omitted (Strang and Fix [15], Ciarlet [7]).

Proceeding as before, we introduce the interpolant $u_{1}$ of $u$ after subtracting (22) from (8). Thus for $v_{h} \in S^{h}$,

$$
\begin{aligned}
\left.\left(q_{h}-q\right) v_{h}\right|_{0} ^{1} & =a_{h}\left(u-u_{I}, v_{h}\right)+a_{h}\left(u_{I}-u_{h}, v_{h}\right) \\
\cdot & -\left(f(u)-f\left(u_{h}\right), v_{h}\right)+\left(a-a_{h}\right)\left(u, v_{h}\right) .
\end{aligned}
$$

The last term in the above expression is the error term for the quadrature formula, and we have the following estimate (Andreev and Lazarov [1], Ciarlet [7])

$$
\left|\left(a-a_{h}\right)\left(u, v_{h}\right)\right| \leqslant C h^{3}\|u\|_{4}\left|v_{h}\right|_{1} .
$$

The term involving $f$ is treated as in Section 3 to yield

$$
\begin{aligned}
\left|\left(f(u)-f\left(u_{h}\right), v_{h}\right)\right| & \leqslant C\left\|u-u_{h}\right\|_{0}\left\|v_{h}\right\|_{0} \\
& \leqslant C h^{3}\|u\|_{3}\left\|v_{h}\right\|_{0} .
\end{aligned}
$$

For the first term, we perform the estimation by freezing the coefficient $a(x)$ at the centroid. Letling $a_{v}^{j}=a\left(\left(x_{j-1}+x_{j}\right) / 2\right)$ when working with element $e_{j}$, we write

$$
a_{h}\left(u-u_{I}, v_{h}\right)=\sum_{j=1}^{N}\left[I_{e_{j}}\left(a_{v}^{j}\left(u-u_{I}\right)^{\prime} v_{h}^{\prime}\right)+I_{e_{j}}\left(a(x)-a_{0}^{j}\right)\left(\left(u-u_{I}\right)^{\prime} v_{h}^{\prime}\right)\right]
$$

and proceed to establish bounds for the $I_{e_{j}}(\cdot)$ terms as follows.

Assuming $u \in H^{4}(\Omega)$, first consider $I_{e_{j}}\left(\left(u-u_{I}\right)^{\prime} v_{h}^{\prime}\right)$ over a reference element. Using standard approximation theory (Ciarlet [7]), norm equivalence on finite dimensional space and the Sobolev embedding theorem, we have

$$
\begin{aligned}
\left|\sum_{k} \hat{w}_{k}\left(\hat{u}-\hat{u}_{I}\right)^{\prime} \hat{v}_{h}^{\prime}\left(\xi_{k}\right)\right| & \leqslant \sum_{k} \hat{w}_{k}\left|\left(\hat{u}-\hat{u}_{I}\right)^{\prime}\right|_{C^{0}(\hat{\Omega})}\left|\hat{v}_{h}^{\prime}\right|_{C^{0}(\hat{\Omega})} \\
& \leqslant \sum_{k} \hat{w}_{k}\left|\hat{u}^{\prime \prime \prime}\right|_{C^{0}(\hat{\Omega})}\left|\hat{v}_{h}^{\prime}\right|_{0, \hat{\Omega}} \\
& \leqslant C \sum_{k} \hat{w}_{k}\left\|\hat{u}^{\prime \prime \prime}\right\|_{1, \hat{\Omega}}\left|\hat{v}_{h}\right|_{1, \hat{\Omega}} \\
& \leqslant C \sum_{k} \hat{w}_{k}\|\hat{u}\|_{\Lambda, \hat{\Omega}}\left|\hat{v}_{h}\right|_{1, \hat{\Omega}} .
\end{aligned}
$$


The last inequality holds since in one dimension, $u \in H^{4}(\Omega)$ implies that $u^{\prime \prime \prime}$ is continuous in $\Omega$. If we let

$$
L \hat{u}=\sum_{k} \hat{w}_{k}\left(\hat{u}-\hat{u}_{I}\right)^{\prime} \hat{v}_{h}^{\prime}\left(\xi_{k}\right)
$$

then from the last estimate, we see that $L$ is a bounded linear operator on $\hat{u} \in H^{4}(\hat{\Omega})$, with

$$
|L \hat{u}| \leqslant\left(\sum_{k} \hat{w}_{k}\right)\|\hat{u}\|_{4, \hat{\Omega}}\left|\hat{v}_{h}\right|_{1, \Omega} .
$$

Since the integration scheme is exact for polynomials of degree 3 , we have $L p=0$ for $p \in \mathbf{P}_{\mathbf{3}}(\hat{\Omega})$. Thus by the Bramble- Hilbert lemma (Ciarlet $[\mathbf{7}]$ ), we may replace that $\|\cdot\|_{4, \Omega}$ norm in (27) by the seminorm $|\cdot|_{4, \Omega}$. Transforming back to the physical elements, we obtain

$$
|L \hat{u}| \leqslant C\left(\sum_{k} w_{k}\right) \cdot h^{3}|u|_{4, \Omega}\left|v_{h}\right|_{1, \Omega} .
$$

For the second term in (26), we have over the reference element

$$
\begin{aligned}
\left|\sum_{k} w_{k}\left(\hat{a}-\hat{a}_{0}\right)\left(\hat{u}^{\prime}-\hat{u}_{I}^{\prime}\right) \hat{v}_{k}\left(\xi_{k}\right)\right| & \leqslant \sum_{k} \hat{w}_{k}\left\|\hat{a}-\hat{a}_{0}\right\|_{C^{0}(\hat{\Omega})}\left\|\left(\hat{u}-\hat{u}_{I}\right)^{\prime}\right\|_{C^{0}(\hat{\mathbf{\Omega}})}\left\|v_{h}^{\prime}\right\|_{C^{\circ}(\hat{\mathbf{\Omega}})} \\
& \leqslant C \sum_{k} \hat{w}_{k}\left\|\hat{a}^{\prime}\right\|_{C^{0}(\hat{\mathbf{n}})}\left\|\hat{u}^{\prime \prime \prime}\right\|_{C^{0}(\hat{\boldsymbol{\Omega}})}\left|v_{h}\right|_{1, \hat{\Omega}} \\
& \leqslant C \sum_{k} \hat{w}_{k}|\hat{a}|_{a, \infty, \hat{\Omega}}\left\|\hat{u}^{\prime \prime \prime}\right\|_{1, \hat{\Omega}}\left|v_{k}\right|_{1, \hat{\Omega}} .
\end{aligned}
$$

And, after transforming back to the physical elements,

$$
\begin{aligned}
& \leqslant C\left[\sum_{k} \hat{w}_{k}\right] h|\hat{a}|_{1, \infty}\left|v_{h}\right|_{1}\left(h^{2}\left\|u^{\prime \prime \prime}\right\|_{0}+h^{3}\left|u^{\prime \prime \prime}\right|_{1}\right), \\
& \leqslant C h^{3}\|u\|_{4}\left|v_{h}\right|_{1} .
\end{aligned}
$$

Combining (28) and (29), we get

$$
\left|a_{h}\left(u-u_{I}, v_{h}\right)\right| \leqslant C h^{3}\|u\|_{4}\left|v_{h}\right|_{1} .
$$

Finally for the second lerm in (23), we observe that for each $v_{h} \in S_{0}^{h}$.

$$
a\left(u, v_{h}\right)-a_{h}\left(u_{h}, v_{h}\right)=\left(f(u)-f\left(u_{h}\right), v_{h}\right),
$$


and so $a_{h}\left(u_{I}-v_{h}, v_{h}\right)=a_{h}\left(u_{I}-u, v_{h}\right)+\left(a_{h}-a\right)\left(u, v_{h}\right)+\left(f(u)-f\left(u_{h}\right), v_{h}\right)$. From (24) to (26) we see that, with the aid of the Poincare inequality,

$$
\left|a_{h}\left(u_{I}-u_{h}, v_{h}\right)\right| \leqslant C h^{3}\|u\|_{4}\left|v_{h}\right|_{1} .
$$

Observing now that $a_{h}(v, v)^{1 / 2}$ is a norm on $S^{h}$ uniformly equivalent to the norm $|v|_{1}$ (Andreev and Lavzrov [1]), we have, upon setting $v_{h}=u_{I}-u_{h}$, the interior superconvergence result,

$$
\left|u_{I}-u_{h}\right|_{1} \leqslant C h^{3}\|u\|_{4}
$$

For $v_{h} \in S^{h}$ but not vanishing on $\partial \Omega$, we have

$$
\begin{aligned}
a_{h}\left(u_{I}-u_{h}, v_{h}\right) & \leqslant a_{h}\left(u_{I}-u_{h}, u_{I}-u_{h}\right)^{1 / 2} a_{h}\left(v_{h}, v_{h}\right)^{1 / 2} \\
& \leqslant C\left|u_{I}-u_{h}\right|_{1}\left|v_{h}\right|_{1}
\end{aligned}
$$

and so from (31),

$$
\left|a_{h}\left(u_{I}-u_{h}, v_{h}\right)\right| \leqslant C h^{3}\|u\|_{4}\left|v_{h}\right|_{1} .
$$

Summarising we have the following results from (24), (25), (31) and (32).

TheOREM 3. Let $q_{h}$ be the approximate flux for the semilinear problem (2) using quadratic elements. Then for any $v_{h} \in S^{h}$,

$$
\left.\left|\left(q-q_{h}\right) v_{h}\right|_{0}^{1}\left|\leqslant C h^{3}\|u\|_{4}\right| v_{h}\right|_{1} .
$$

Further, by settling $v_{h}=x$ and $1-x$ respectively, we have

Corollary 3. The approximate flux $q_{h}$ for (2) using quadratic element is third order accurate, that is

$$
\left|\left(q-q_{h}\right)(0)\right|+\left|\left(q-q_{h}\right)(1)\right| \leqslant C h^{3}\|u\|_{4} .
$$

When $f(u)$ is linear, the above corollary yields a suboptimal estimate as the optimal error is $O\left(h^{4}\right)$ (see Wheeler [20] and Dupont [10]). One obvious factor that seems to contribute to the lower order of convergence is the use of numerical quadrature in our calculation of the finite element approximations. However, even if we assume all quadralures are evaluated exactly, the estimate remains suboptimal when compared with the linear case. The major advantage of our approach is that extension to higher dimensional problems is relatively easy compared with the other methods used in deriving optimal results for linear problems in one dimension. 
Actually Theorem 3 may also be shown to hold if we choose the two point Gauss rule as our quadrature formula. To show that (24) and (28) hold in this case we need to verify that for $p \in \mathbf{P}_{3}(\hat{\Omega}), L p=0$. For $p=\xi^{3}, p_{I}=\xi$. Thus $L p=\left.\sum_{k=1}^{2}\left(\xi^{3}-\xi\right)^{\prime} v_{h}^{\prime}\right|_{ \pm 1 / \sqrt{3}}=$ $\sum_{k=1}^{2}\left[3[ \pm 1 / \sqrt{3}]^{2}-1\right] v_{h}^{\prime}[ \pm 1 / \sqrt{3}]=0$.

For higher order elements, the line of argument is similar. For example, for cubic elements, we may employ the standard three point Gauss rule to obtain an $O\left(h^{4}\right)$ estimate for the flux error. For quartic and higher order elements, we must also be careful in selecting the interpolation points that define $u_{1}$. For example, we use the points $0, \pm \sqrt{3 / 7}, \pm 1$ in the interpolation process when quarlic elements are used (see Zlamal [21]).

\section{Parabolic boundary value problem}

The results obtained in the previous sections may also be extended to parabolic problems. We first study the semilinear problem

$$
\begin{gathered}
u_{t}=\left(a(x) u_{x}\right)_{x}+f(u) \\
u(0, t)=0, u(1, t)=0 \\
u(x, 0)=u_{0}(x)
\end{gathered}
$$

with $a(x)$ and $f(u)$ possessing properties described in Section 2. We further assume that the initial data $u_{0}(x)$ is sufficiently smooth

In weak form, we have

$$
\left(u_{t}, v\right)+\left(a u_{x}, v_{x}\right)=(f(u), v) \text { for } t \geqslant 0
$$

where $v=v(x)$ is a smooth function which vanishes at $x=0,1$ and $(v, w)$ denotes as before the inner product $\int_{0}^{1} v w d x$ in $L^{2}(0,1)$. The semidiscrete finite element approximation $u_{h}(t)$ may then be computed by solving the following problem:

$$
\left(u_{h t}, v_{h}\right)+\left(a u_{h x}, v_{h x}\right)=\left(f\left(u_{h}\right), v_{h}\right) \text { for all } v_{h} \text { in } S^{h}, t \geqslant 0
$$

with the initial condition given by $u_{h}(0)=u_{0 h}$

$$
\left(a u_{0 h x}, v_{h x}\right)=\left(a u_{0 x}, v_{h x}\right) \text { for all } v_{h} \text { in } S_{0}^{h}
$$

Introducing the elliptic projection $P_{1}$ onto $S_{0}^{h}$ for $u \in H_{0}^{1}(\Omega)$ via

$$
\left(a\left(P_{1} u\right)_{x}, v_{h_{x}}\right)=\left(a u_{x}, v_{h_{x}}\right) \text { for all } v_{h} \text { in } S_{0}^{h}
$$


we may study the error $u_{h}-u$ in terms of $u_{h}-P u \equiv \theta$ and $P_{1} u \equiv \rho$. Note that $u_{0 h}=$ $\rho_{1} u_{0}$. Applying the standard techniques (Wheeler [19], Thomé [16]), in estimating $\theta$ and $\rho$ while taking some care in treating the nonlinear $f(u)$ term, we have the following estimates:

$$
\begin{gathered}
\|\rho(t)\|_{0}+h\left\|\rho_{x}(t)\right\|_{0} \leqslant C h^{r}\left\{\left\|u_{0}\right\|_{r}+\int_{0}^{t}\left\|u_{t}\right\|_{r} d s\right\}, t>0 \\
\frac{d}{d t}\|\theta\|_{0} \leqslant\left\|\rho_{t}\right\|_{0}+\gamma\|\rho\|_{0} \leqslant C h^{r}\left\{\left\|u_{t}\right\|_{r}+\int_{0}^{t}\left\|u_{t}\right\|_{r} d s\right\}
\end{gathered}
$$

where $r-1$ is the degree of the finite element space. Furthermore, by setting $\chi=\theta_{t}$ in the equation

$$
\left(\theta_{t}, \chi\right)+\left(a \theta_{x}, \chi_{x}\right)=-\left(\rho_{t}, \chi\right)+\left(f\left(u_{h}\right)-f(u), \chi\right) \text { for all } \chi \text { in } S_{0}^{h},
$$

we obtain

$$
\frac{d}{d t}\left(a \theta_{x}, \theta_{x}\right) \leqslant\left\|\rho_{t}\right\|_{0}^{2}+2 \gamma^{2}\|\rho\|_{0}^{2}+2 \gamma^{2}\|\theta\|_{0}^{2}
$$

which gives, after integration and using (4) and the fact from (36) that $\theta(0)=0$, the interior superconvergence result for $\theta_{x}$ :

$$
\left\|\theta_{x}(t)\right\|_{0} \leqslant C\left(t,\left\|u_{t}\right\|_{r},\left\|u_{0}\right\|_{r}\right) h^{r}, t \geqslant 0
$$

The flux $q(t)$ satisfies the relation

$$
-\left.q v\right|_{0} ^{1}=\left(u_{t}, v\right)+a(u, v)-(f(u) v) \text { for all } v \text { in } H_{0}^{1}(\Omega)
$$

and so the approximate flux $q_{h}$ is defined via

$$
-\left.q_{h} v_{h}\right|_{0} ^{1}=\left(u_{h t}, v_{h}\right)+a\left(u_{h}, v_{h}\right)-f\left(u_{h}, v_{h}\right) \text { for all } v_{h} \text { in } S^{h} .
$$

Thus subtracting these expressions, and introducing the elliptic projection $P_{1}$, we have

$$
\begin{array}{r}
\left.\left(q_{h}-q\right) v_{h}\right|_{0} ^{1}=\left(\rho_{t}, v_{h}\right)+\left(\theta_{t}, v_{h}\right)+a\left(\rho, v_{h}\right)+a\left(\theta, v_{h}\right) \\
+\left(f(u)-f\left(u_{h}\right), v_{h}\right) \text { for all } v_{h} \text { in } S^{h} .
\end{array}
$$

From (38) we have $\left|\left(\rho_{\mathrm{t}}, v_{h}\right)+\left(\theta_{t}, v_{h}\right)\right| \leqslant C h^{r}\left\|u_{t}\right\|_{r}\left\|v_{h}\right\|_{0}$. The estimate (39) gives us the bound $\left|a\left(\theta, v_{h}\right)\right| \leqslant C\left(\|u\|_{r}\right) h^{r}\left|v_{h}\right|$. The last term is bounded by $C\left\|u-u_{h}\right\|_{0}\left\|v_{h}\right\|_{0}$ which in turn may be bounded by applying (37), (38) to yield $\left|\left(f(u)-f\left(u_{h}\right), v_{h}\right)\right| \leqslant$ $C\left(\|u\|_{r},\left\|u_{0}\right\|_{r}\right) h^{r}\left\|v_{h}\right\|_{0}$. Here $C(\|\cdot\|)$ signifies that the constant is dependent on the norm $\|\cdot\|$. To treat the term $a\left(\rho, v_{h}\right)$, we introduce the interpolant $u_{I}(t)$ of $u(t)$ at 
time $t$ in $S^{h}$. Note here that $a\left(\rho, v_{h}\right)=0$ only for $v_{h} \in S_{0}^{h}$, and not necessarily so for a general $v_{h}$ in $S^{h}$. Thus we write

$$
a\left(\rho, v_{h}\right)=a\left(P_{1} u-u_{I}, v_{h}\right)+a\left(u-u_{I}, v_{h}\right)
$$

and proceed to estimate the terms on the right hand side.

For the second term, we may proceed as in either the case of linear elements (12) or quadratic elements (30), while taking into account the effect of numerical integration in the latter case, to obtain the bound

$$
\left|a\left(u-u_{1}\right) v_{h}\right| \leqslant C h^{r}\|u\|_{2(r-1)}\left|v_{h}\right|_{1}, r=2,3 .
$$

Since both $P_{1} u$ and $u_{I}$ are in $S_{0}^{h}$, we make use of the coercivity and boundedness of the bilinear form $a(\cdot, \cdot)$ on $V$ and $(40)$ to extimate $a\left(P_{1} u-u_{I}, v_{h}\right)$. Since

and

$$
\begin{aligned}
a_{0}\left|P_{1} u-u_{I}\right|^{2} & \leqslant a\left(P_{1} u-u_{I}, P_{1} u-u_{I}\right) \\
& =a\left(u-u_{I}, P_{1} u-u_{I}\right) \\
& \leqslant C h^{r}\|u\|_{2(r-1)}\left|P_{1} u-u_{I}\right|_{1}, r=2,3
\end{aligned}
$$

we have $\quad a\left(P_{1} u-u_{I}, v_{h}\right) \leqslant C h^{r}\|u\|_{2(r-1)}\left|v_{h}\right|_{1}, r=2,3$.

Combining the results in this section we have

TheOREM 4. Let $q$ be the flux defined in (40) and $q_{h}$ the approximate flux defined in (41) and let $r-1$ be the degree of the finite element with $r=2$ or 3 . Then for all $v_{h} \in S^{h}$ and $t \geqslant 0$,

$$
\left.\left|\left(q-q_{h}\right) v_{h}\right|_{0}^{1}\left|\leqslant C h^{r}\right| v_{h}\right|_{1}
$$

where $C$ is dependent on various norms of $u$ and $u_{0}$.

Selting $v_{h}=x$ and $1-x$ respectively we have

Corollary 4. The error $\left|\left(q-q_{h}\right)(0)\right|+\left|\left(q-q_{h}\right)(1)\right|$ of the parabolic problem (33) is $O\left(h^{r}\right), r=2,3$.

\section{REFERENCES}

[1] A.B. Andreev and R.D. Lazarov, 'Superconvergence of the gradient for quadratic triangular finite elements', Numer. Methods Partial Differential Equations 4 (1988), 15-31.

[2] J.W. Barrett and C.M. Elliott, 'Total flux estimates for a finite-element approximation of elliptic equations', I.M.A. J. Numer. Anal. 7 (1987), 129-148.

[3] F.E. Browder, 'Existence and uniqueness theorems for solutions of nonlinear boundary value problems', Proc. Sympos. Appl. Math. 17 (1065), 24-49. 
[4] G.F. Carey, 'Derivative calculations from finite element solutions', J. Comp. Meth. Applied Mech. Eng. 35 (1982), 1-4.

[5] G.F. Carey, S.-S. Chow and M. Seager, 'Approximate boundary-flux calculations', J. Comp. Meth. Applied Mech. Eng. 50 (1985), 107-120.

[0] S.-S. Chow, 'Finite element error estimates for non-linear elliptic equations of monotone type', Numer. Math. 54 (1989), 373-393.

[7] P.E. Ciarlet, The finite element method for elliptic problems (North-Holland, Amsterdam, 1978).

[8] J. Douglas Jr. and ' $\mathrm{r}$. Dupont, 'Galerkin approximations for the two point boundary problems using continuous piecewise polynomial spaces', Numer. Math. 22 (1974), 99-109.

[9] J. Douglas, T. Dupont and M.F. Wheeler, 'A Galerkin procedure for approximating the flux on the boundary for elliptic and parabolic boundary value problems', RAIRO Anal. Numer. 2 (1974), 47-59.

[10] T. Dupont, 'A mified theory of superconvergence for Galerkin methods for two-point boundary probleins', SIAM J. Numer. Anal. 13 (1976), 362-368.

[11] R. Clowinski and A. Marrocco, 'Analyse numérique du champ magnétique d'un alternateur par élements finis et sur-relaxation ponctuelle non liéaire', J. Comp. Meth. Applied Mech. Eng. 3 (1974), 55-85.

[12] R.D. Lazarov,A.I. Pehlivanov, S.-S. Chow and G.F. Carey, 'Superconvergence analysis of boundary-flux calculations', (in preparation).

[13] M. Krìžek and P. Neittaanıäki, 'On superconvergence techniques', Acta. Appl. Math. $\theta$ (1987), 175-198.

[14] M.A. Noor and J.R. Whiteman, 'Error bounds for finite element solutions of mildly nonlinear elliptic boundary value problems', Numer. Math. 26 (1976), 107-116.

[15] G. Strang and G.J. Fix, An analysis of the finite element method (Prentice-Hall, Englewood Cliffs, 1971).

[16] V. Thomée, Galerkin finite element methods for parabolic problems: Lecture Notes in Math. 1054 (Springer-Verlag, Berlin, 1984).

[17] M.M. Vainberg, Variational method and method of monotone operators in the theory of nonlinear equations (Halsted Press, John Wiley, New York, 1973).

[18] J.A. Wheeler, 'Simulation of heat transfer from a warm pipeline buried in permafrost', 74th National Meeting of American lnstitute of Chemical Engineers, New Orleans.

[19] M.F. Wheeler, 'A priori $L_{2}$ error estimates for Galerton approximations to parabolic partial differential equations', SIAM J. Numer. Anal. 10 (1973), 723-759.

[20] M.F. Wheeler, 'A Galerkin procedure for eslimating the flux for two-point boundary value problems', SIAM J. Numer. Anal. 11 (1974), 764-768.

[21] M. Alámal, 'Some superconvergence results in the finite element method', in Mathematical Aspects of Finite Element Methods: Lecture Notes in Math. 606, pp. 363-362 (Springer-Verlag, Berlin, 1977).

Department of Mathematics

University of $\mathrm{W}$ yoming

I,aramie

United States of America
Bulgarian Academy of Sciences Institute of Mathematics

Sotia

Bulgaria 